\title{
Can MRI Replace CT in Evaluating Semicircular Canal Dehiscence?
}

\author{
P. Browaeys, T.L. Larson, M.L. Wong, and U. Patel
}

\section{ABSTRACT}

BACKGROUND AND PURPOSE: Patients with symptoms of semicircular canal dehiscence often undergo both CT and MR imaging. We assessed whether FIESTA can replace temporal bone CT in evaluating patients for SC dehiscence.

MATERIALS AND METHODS: We retrospectively reviewed 112 consecutive patients (224 ears) with vestibulocochlear symptoms who underwent concurrent MR imaging and CT of the temporal bones between 2007 and 2009. MR imaging protocol included a FIESTA sequence covering the temporal bone (axial 0.8-mm section thickness, 0.4-mm spacing, coronal/oblique reformations; 41 patients at 1.5T, 71 patients at 3T). CT was performed on a 64-row multidetector row scanner (0.625-mm axial acquisition, with coronal/oblique reformations). Both ears of each patient were evaluated for dehiscence of the superior and posterior semicircular canals in consensual fashion by 2 neuroradiologists. Analysis of the FIESTA sequence and reformations was performed first for the MR imaging evaluation. CT evaluation was performed at least 2 weeks after the MR imaging review, resulting in a blinded comparison of CT with MR imaging. CT was used as the reference standard to evaluate the MR imaging results.

RESULTS: For SSC dehiscence, MR imaging sensitivity was 100\%, specificity was $96.5 \%$, positive predictive value was $61.1 \%$, and negative predictive value was $100 \%$ in comparison with CT. For PSC dehiscence, MR imaging sensitivity was $100 \%$, specificity was $99.1 \%$, positive predictive value was $33.3 \%$, and negative predictive value was $100 \%$ in comparison with CT.

CONCLUSIONS: MR imaging, with a sensitivity and negative predictive value of $100 \%$, conclusively excludes SSC or PSC dehiscence. Negative findings on MR imaging preclude the need for CT to detect SC dehiscence. Only patients with positive findings on MR imaging should undergo CT evaluation.

ABBREVIATIONS: $\mathrm{Cl}$ = confidence interval; FIESTA = fast imaging employing steady-state acquisition; $\mathrm{PSC}=$ posterior semicircular canal; $\mathrm{SC}=$ semicircular canal; SSC $=$ superior semicircular canal

$\mathbf{S}_{\mathrm{w}}^{\mathrm{w}}$ uperior semicircular canal dehiscence was first described by Minor et al. ${ }^{1}$ Patients with SSC dehiscence classically present with symptoms of both auditory and vestibular dysfunction. Ves-

Received February 11, 2012; accepted after revision September 10.

From the Department of Radiology (P.B.), University Hospital, Lausanne, Switzerland; Department of Radiology (P.B.), Montchoisi Clinic, Lausanne, Switzerland; University of Washington (P.B.), Seattle, Washington; Seattle Radiologists (T.L.L., U.P.), Seattle, Washington; and Washington Otology/Neurotology Group (M.L.W.), Seattle, Washington.

Paper previously presented at: Annual Meeting of the American Society of Neuroradiology and Neuroradiology Education and Research Foundation Symposium, May 15-20, 2010; Boston, Massachusetts (recipient of the Head and Neck award), and at Annual Meeting of the American Society of Head and Neck Radiology, October 6-10, 2010, Houston, Texas (recipient of the Radiologist-in-Training award).

Please address correspondence to Patrick Browaeys, MD, MHS, Department of Radiology, University Hospital of Lausanne, 46 Rue du Bugnon-BH07-RAD CHUV, CH-1011 Lausanne, Switzerland; and Department of Radiology, Montchoisi Clinic; Genolier Swiss Medical Network, 10 Chemin des Allinges, CH-1006 Lausanne, Switzerland; e-mail: patrick.browaeys@skynet.be

-- Indicates open access to non-subscribers at www.ajnr.org

http://dx.doi.org/10.3174/ajnr.A3459 tibular symptoms include the phenomena of Tullio $^{2}$ (vertigo and/or nystagmus induced by loud noise) and Hennebert ${ }^{3}$ (Reference 3: Krombach et al) (vertigo and/or nystagmus induced by pressure in the external auditory canal). Auditory symptoms include hearing loss, typically low-frequency.

Normally the pressure wave generated by sound is transmitted via the ossicles to the oval window and dissipated through the round window. SSC dehiscence results in the creation of a "third" window, allowing the sonic pressure wave to be dissipated through this third window. This nonphysiologic sonic pressure wave can stimulate vestibular afferents, resulting in vestibular symptoms. ${ }^{4}$ Patients with SSC dehiscence can present with myriad other symptoms, however, such as dizziness, chronic imbalance, and autophony. ${ }^{5}$ Patients with SSC dehiscence have been misdiagnosed as having otosclerosis, patulous eustachian tube, Meniere disease, benign paroxysmal vertigo, perilymph fistula, and psychiatric disorders. ${ }^{6}$ Dehiscence of the posterior semicircular canal is a less common entity and produces symptoms similar to those of SSC dehiscence. ${ }^{7,8}$ 

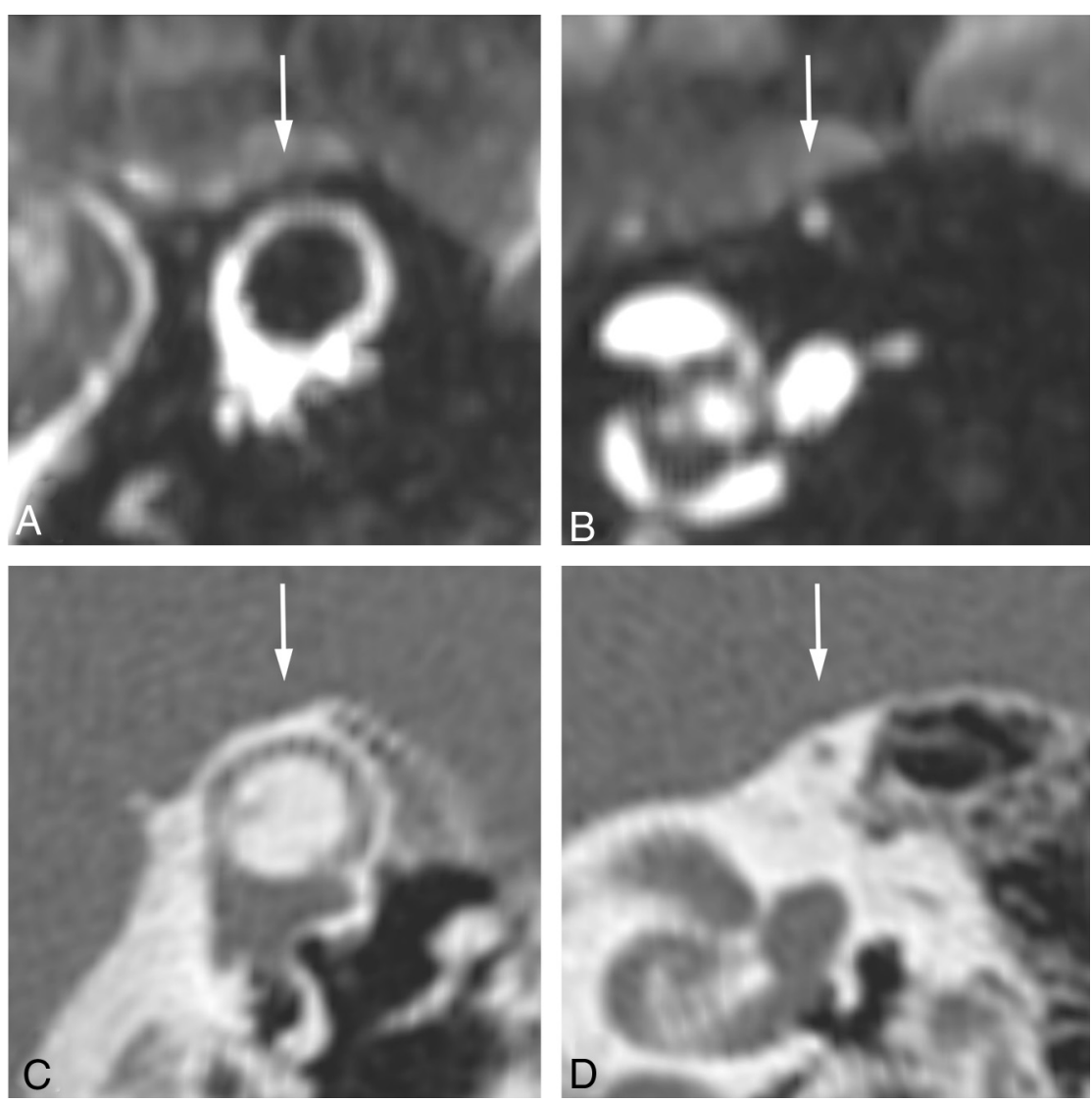

FIG 1. Normal-appearing SSC (left ear) without dehiscence in a 17-year-old boy. FIESTA images show the SSC in a long-axis plane $(A)$ and in a short-axis plane (B): the bright SC content corresponds to both perilymph and endolymph, and bone coverage has low signal intensity (arrows). Corresponding CT images of the same patient clearly demonstrate bone coverage (arrows) in the long-axis $(C)$ and in the short-axis $(D)$ planes.

Table 1: MRI/CT detection of the superior semicircular canal dehiscence ${ }^{a}$

\begin{tabular}{lcc}
\hline SSC (213 Canals, 11 Excluded) & Dehiscence on CT (No.) & No Dehiscence on CT (No.) \\
\hline Dehiscence on MRI (1.5T/3T) & $11(5 / 6)$ & $7(2 / 5)$ \\
No dehiscence on MRI (1.5T/3T) & $0(0 / 0)$ & $195(72 / 123)$ \\
\hline
\end{tabular}

${ }^{a}$ Detailed results for 1.5T and 3T are displayed in parentheses as follows: (1.5T/3T). Of the 224 SSCs in 112 patients, 11 were excluded because of artifacts on CT and/or MRI. In the remaining 213 canals, 11 of the $18(11+7)$ SSC dehiscence cases seen on FIESTA were confirmed on CT. When there was no SSC dehiscence on MRI, there was no SSC dehiscence seen on $\mathrm{CT}$.

When presented with a patient manifesting the above symptoms, neurotologists often order both MR imaging and CT for further evaluation, MR imaging to exclude vestibular schwannoma and other tumors of the temporal bone, brain stem, and posterior fossa and to assess brain stem infarct, arterial dissection, and demyelinating disease; and CT to assess the integrity of the semicircular canals, rule out otosclerosis, and evaluate the middle ear for diseases such as cholesteatoma.

Patients and referring physicians are becoming more cognizant of the cost of performing 2 imaging studies, and many insurers are reluctant to reimburse for 2 imaging studies of the same body part. Additionally, there is added awareness of the risks of CT radiation. ${ }^{9}$

Thin-section heavily T2-weighted imaging sequences such as FIESTA and constructive interference in steady state MR imaging are commonly used in imaging patients with vertigo, and their utility in evaluating cranial nerve pathology has been proved. ${ }^{10,11}$
The FIESTA sequence provides highcontrast definition between fluid and bone, and its high spatial resolution results in an exquisite anatomic definition of the membranous labyrinth. Motion artifacts are minimized by a balanced acquisition; hence, CSF pulsation artifacts are minimized. ${ }^{12}$

We hypothesized that the absence of a hypointense layer between fluid in the semicircular canal and CSF on FIESTA should indicate an absence of bony coverage, or dehiscence. Conversely, the presence of a hypointense layer should indicate bone coverage and the absence of dehiscence. We undertook this study to determine whether FIESTA can replace CT of the temporal bone in evaluating patients for SC dehiscence.

\section{MATERIALS AND METHODS Design of the Study}

We retrospectively reviewed 112 consecutive MR imaging and CT scans of the temporal bones obtained between October 2007 and October 2009 in 112 patients (39 men and 73 women, with 1 woman having been examined twice within a 14-month interval; mean age, $51 \pm 14$ years; range, 17-85 years) referred for symptoms such as vertigo, dizziness, tinnitus, or hearing loss. The CT and MR imaging examinations were performed concurrently, at the request of the neurotology service.

The 112 examinations covering the bilateral temporal bones allowed the evaluation of 224 ears and the corresponding SSC and PSC of each ear. Because the lateral semicircular canal does not have an interface with CSF, it was not analyzed. MR imaging examinations were performed at $1.5 \mathrm{~T}$ in 41 patients and at $3 \mathrm{~T}$ in 71 patients. Scans were assigned a 10-digit reference number, and patient identifiers were removed for blinding. Analysis of the FIESTA sequence was performed first, for all patients and on both ears, with the MR imaging data being sorted by the date of acquisition. Analysis of CT images was then performed at least 2 weeks later, for all patients and on both ears. The data were sorted by the reference number, resulting in blinded comparison of FIESTA with CT.

\section{Technical Considerations of MR Imaging and CT Acquisitions}

All MR imaging scans included an axial high-resolution FIESTA acquisition covering both temporal bones; additional sequences dedicated to the brain were acquired but not analyzed for the purpose of this study. T2* gradient-echo localizers in 3 orthogonal planes were used to prescribe FIESTA acquisition through the 
Table 2: Data distribution between 1.5T and 3T subgroups

\begin{tabular}{|c|c|c|c|c|}
\hline & $\begin{array}{c}\text { Dehiscence } \\
\text { CT and MRI } \\
\text { (No.) }\end{array}$ & $\begin{array}{l}\text { Dehiscence } \\
\text { CT Only } \\
\text { (No.) }\end{array}$ & $\begin{array}{l}\text { Dehiscence } \\
\text { MRI Only } \\
\text { (No.) }\end{array}$ & $\begin{array}{c}\text { No Dehiscence } \\
\text { on Both } \\
\text { CT and MRI } \\
\text { (No.) }\end{array}$ \\
\hline \multicolumn{5}{|l|}{ SSC } \\
\hline $1.5 \mathrm{~T}$ & 5 & 0 & 2 & 72 \\
\hline 3T & 6 & 0 & 5 & 123 \\
\hline & \multicolumn{4}{|c|}{$P$ value for distribution comparison (Fisher exact test) $=.80^{\mathrm{a}}$} \\
\hline \multicolumn{5}{|l|}{ PSC } \\
\hline $1.5 \mathrm{~T}$ & 1 & 0 & 0 & 79 \\
\hline $3 \mathrm{~T}$ & 0 & 0 & 2 & 133 \\
\hline & \multicolumn{4}{|c|}{$P$ value for distribution comparison (Fisher exact test) $=.29^{\mathrm{a}}$} \\
\hline
\end{tabular}

${ }^{a} \mathrm{~A} P$ value $\geq .05$ means that the hypothesis of equality between the 2 distributions is not rejected by the Fisher exact test.
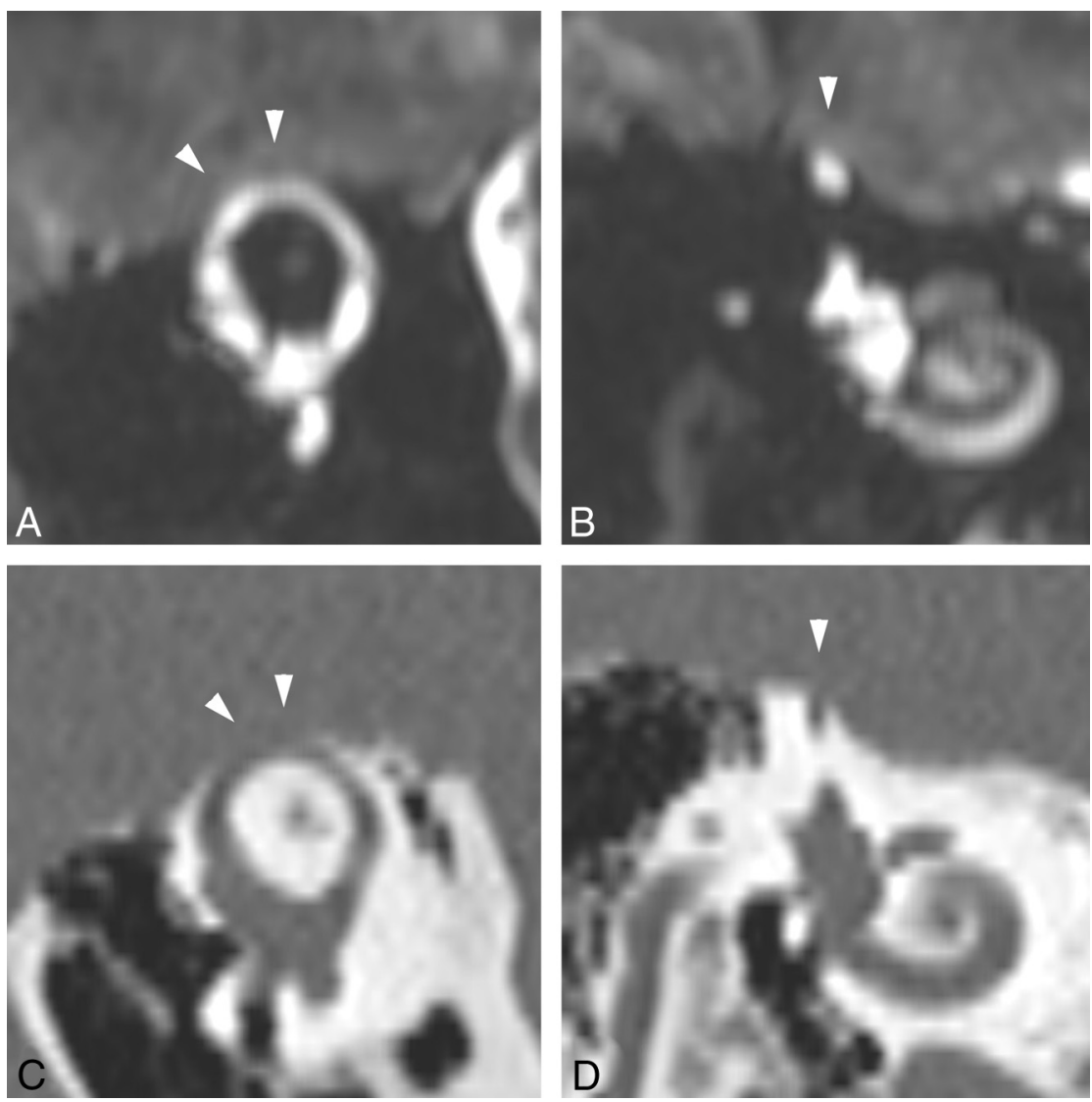

FIG 2. A 35-year-old woman with bilateral tinnitus and hearing loss. FIESTA images of the right SSC in the long-axis $(A)$ and short-axis $(B)$ planes show the absence of a low-signal-intensity rim (arrowheads) between the canal and the middle cranial fossa, characteristic of SSC dehiscence. Corresponding CT images ( $C$ and $D$ ) confirm the absence of bone between the SSC and the middle cranial fossa (arrowheads). In this case, dehiscence in the long axis was measured at $5.1 \mathrm{~mm}$ on FIESTA and $3.6 \mathrm{~mm}$ on CT.

labyrinths. An 8-channel head coil was used on both 1.5T and 3T scanners (Signa HDX; GE Healthcare, Milwaukee, Wisconsin). Specific 1.5T FIESTA acquisition parameters were the following: TR, $5.504 \mathrm{~ms}$; TE, $1.96 \mathrm{~ms}$; gradient maximal amplitude, $33 \mathrm{mT} / \mathrm{m}$; gradient rise time, $276 \mu \mathrm{s}$; gradient slew rate, $120 \mathrm{Tm}^{-1} \mathrm{~s}^{-1}$; section thickness, $0.8 \mathrm{~mm}$; section spacing, $0.4 \mathrm{~mm}$; NEX, 4; flip angle, $55^{\circ}$; acquisition matrix, $256 \times 256$ with a $16-\mathrm{cm}$ square FOV leading to a pixel size of $0.3125 \times 0.3125 \mathrm{~mm}$ in the axial plane. Fifty-two sections covering $20.8 \mathrm{~mm}$ were obtained in 3 minutes 18 seconds.
Specific 3T FIESTA acquisition parameters were the following: TR, $4.9 \mathrm{~ms}$; TE, $2.4 \mathrm{~ms}$; gradient maximal amplitude, $40 \mathrm{mT} / \mathrm{m}$; gradient rise time, 267 $\mu \mathrm{s}$; gradient slew rate, $150 \mathrm{Tm}^{-1} \mathrm{~s}^{-1}$; section thickness, $0.8 \mathrm{~mm}$; section spacing, $0.4 \mathrm{~mm}$; NEX, 1; flip angle, $37^{\circ}$; acquisition matrix, $256 \times 256$ with an $18-\mathrm{cm}$ square FOV leading to a pixel size of $0.352 \times 0.352 \mathrm{~mm}$ in the axial plane. Eighty-four sections covering $33.6 \mathrm{~mm}$ were obtained in 1 minute 57 seconds.

CT was performed on a helical 64section scanner (LightSpeed VCT; GE Healthcare). All patients were examined in the supine position, and the gantry was not tilted. Images were acquired by using incremental axial scans, with a section thickness of $0.625 \mathrm{~mm}$ and no gap. Acquisition parameters were $190 \mathrm{~mA}$ and $140 \mathrm{kV}$. The images were reconstructed from the raw data with a $512 \times$ 512 matrix and a $10-\mathrm{cm}$ FOV, leading to a $0.195 \times 0.195 \mathrm{~mm}$ pixel size in the plane of acquisition. A bone sharpening filter (Bone Plus, GE Healthcare) was used, with edge enhancement. The default-viewing parameters used were $4000 \mathrm{HU}$ for window and $400 \mathrm{HU}$ for level; these values could be adjusted if needed during the review process. Sagittal long-axis and $90^{\circ}$ orthogonal shortaxis views in both SSC and PSC were performed as a standard by technologists for MR imaging and CT examinations, following the method described by Branstetter et al and Belden et al ${ }^{13,14}$ (example shown in Fig 1), and were included in the dataset for analysis.

\section{Data Analysis}

SSC or PSC dehiscence assessment was done by consensus between 2 neuroradiologists (P.B., U.P.). This was achieved by reviewing the axial source images and reformations. If needed, additional multiplanar reformations were processed by the reviewing neuroradiologists from the axial source data on an Advantage Windows workstation (AWsuite 2.0.6.5.1z, Volume Viewer 2; GE Healthcare). For each patient, We analyzed both ears one after the other, with access to clinical data. Dehiscence was measured in millimeters on the long-axis view of the affected semicircular canal. The boundaries of the dehiscence were defined by highattenuation bone margins on CT and low-signal bone margins on FIESTA. The FIESTA sequence was used solely for MR imaging evaluation of the SC.

AJNR Am J Neuroradiol 34:1421-27 Jul 2013 www.ajnr.org 

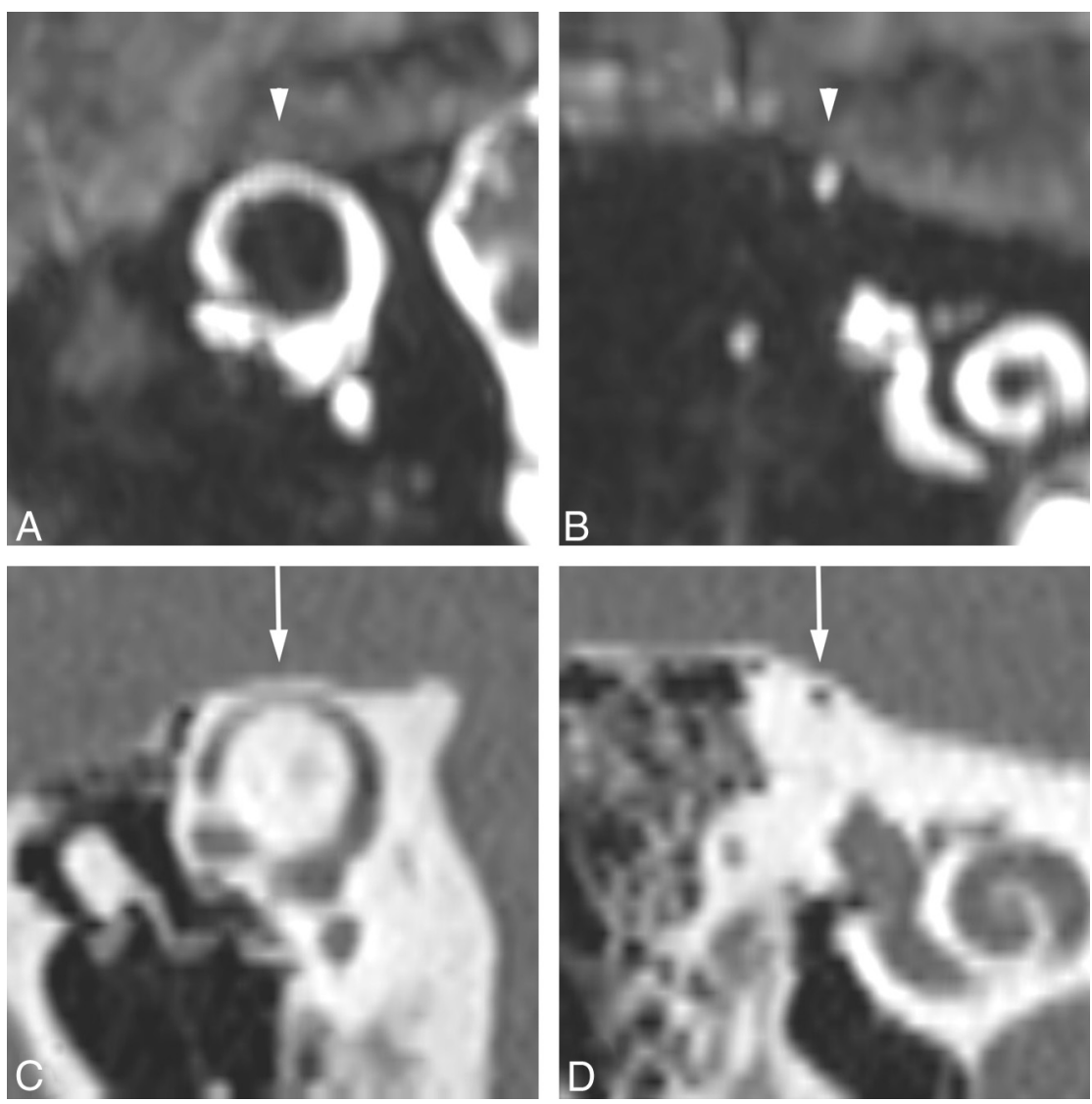

FIG 3. A 46-year-old woman with right-sided hearing loss. On FIESTA, absence of a low signal rim along the superior aspect of the right SSC (arrowheads) on both its long axis (A) and short axis (B) suggests SSC dehiscence. Corresponding $\mathrm{CT}(C$ and $D)$ shows thin but clearly visible bone covering the right SSC (arrows).

Table 3: MRI/CT detection of posterior semicircular canal dehiscence ${ }^{a}$

\begin{tabular}{lcc}
\hline PSC (215 Canals, 9 Excluded) & Dehiscence on CT (No.) & No Dehiscence on CT (No.) \\
\hline Dehiscence on MRI (1.5T/3T) & $1(1 / 0)$ & $2(0 / 2)$ \\
No dehiscence on MRI & $0(0 / 0)$ & $212(79 / 133)$ \\
\hline
\end{tabular}

${ }^{a}$ Detailed results for 1.5T and 3T are displayed in parentheses as follows: (1.5T/3T). Of the 224 PSCs in 112 patients, 9 were excluded because of artifacts on CT and/or MRI. In the remaining 215 canals, 1 of the $3(1+2)$ cases of SSC dehiscence seen on FIESTA was confirmed on CT. When there was no PSC dehiscence on MRI, there was no PSC dehiscence seen on $\mathrm{CT}$.

Poor image data from artifacts, motion, and/or noninclusion of pertinent labyrinth anatomy precluded characterization of dehiscence in 20 semicircular canals (11 SSCs and 9 PSCs respectively). Of these 20 SCs, 4 SCs (in 3 patients; 3 SSCs and 1 PSC) had artifacts on both MR imaging and CT. The remaining 16 SCs (in 6 patients; 8 SSCs and 8 PSCs) were not characterizable on MR imaging, though they were clearly seen on CT.

Sensitivity, specificity, positive predictive value, and negative predictive value were calculated for FIESTA results, by using the CT data as the standard reference.

\section{Statistical Analysis}

Statistical analysis was performed on PSPP (Release 0.6.0; SAS, Cary, North Carolina). We calculated confidence intervals for dehiscence assessment separately by using the conservative exact binomial-based method of Clopper-Pearson, considering results distribution. The standard Student $t$ test was applied to compare measurements. The Fisher exact test was used to compare datasets distribution.

\section{RESULTS \\ SSC Dehiscence}

Of 224 possible SSCs, 213 were included in the analysis; 11 SSCs in 9 patients were excluded because of poor image quality due to motion or other artifacts on MR imaging and/or CT. Table 1 summarizes the SSC dehiscence detection by MR imaging and CT. Because there was no statistically significant difference $(P=.8)$ between the $1.5 \mathrm{~T}$ and $3 \mathrm{~T}$ datasets (Table 2), we merged the two. The incidence of SSC dehiscence demonstrated on CT was $5.2 \%$ ( 11 cases of SSC dehiscence in 7 patients, 4 of whom had bilateral SSC dehiscence). The incidence of SSC dehiscence demonstrated on MR imaging was $8.5 \%$ (18 cases of SSC dehiscence in 12 patients, 6 of whom had bilateral SSC dehiscence).

MR imaging depicted all cases of SSC dehiscence seen on CT; therefore, the sensitivity was $100 \%$ (95\% CI, 71.5\%$100 \%)$. Specificity was $96.5 \%$ (95\% CI, 93\%-98.6\%). An example of positive SSC dehiscence on MR imaging and CT can be seen in Fig 2. There were 7 falsepositive SSC dehiscence cases on MR imaging compared with CT (Fig 3). The positive predictive value was $61.1 \%$ (95\% CI, 35.7\%-82.7\%). Because there were no instances of SSC dehiscence missed on MR imaging, the negative predictive value was $100 \%$ (95\% CI, $98.1 \%-100 \%)$.

\section{PSC Dehiscence}

Of 224 possible PSCs, 215 were included in the analysis; 9 PSCs in 7 patients were excluded for the same reasons described for SSC. Table 3 summarizes PSC dehiscence detection on MR imaging and CT. Because there was no statistically significant difference $(P=.29)$ between 1.5T and 3T datasets (Table 2), we merged the two. The incidence of PSC dehiscence on CT was $0.5 \%$ ( 1 PSC dehiscence in 1 patient, Fig 4). The incidence of PSC dehiscence on MR imaging was $1.4 \%$ (3 PSC dehiscence cases in 3 patients). MR imaging depicted the single PSC dehiscence seen on CT; therefore, the sensitivity was $100 \%$ (95\% CI, 2.5\%-100\%). The specificity was $99.1 \%$ (95\% CI, 96.7-99.9\%). There were 2 false-positive PSC dehiscence cases on MR imaging compared with CT; therefore, the positive predictive value was $33.3 \%$ (95\% CI, 0.8\%-90.6\%). Because there were no PSC dehiscence cases missed on MR imaging compared with CT, the negative predictive value was 100\% (95\% CI, 98.3\%-100\%).

\section{Aggregate Data}

Of the 448 SCs (SSC and PSC) in 112 patients, CT showed 12 cases of SC dehiscence ( 8 patients) in 444 canals analyzable; 4 

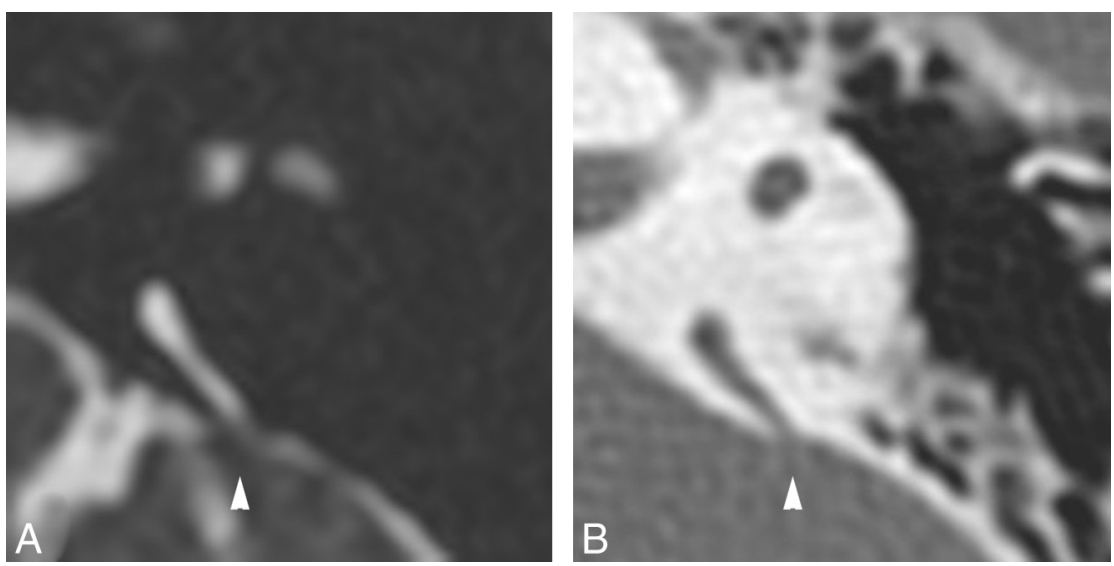

FIG 4. A 43-year-old man with muffled hearing on both sides. Axial FIESTA $(A)$ and CT (B) images of the left temporal bone. There is absence of a low-signal rim between the PSC and the cerebellopontine angle cistern ( $A$, arrowhead) with corresponding absence of bone coverage ( $B$, arrowhead) in this patient with PSC dehiscence.

Table 4: MRI detection of patients with SC dehiscence

\begin{tabular}{lcc}
\multicolumn{1}{c}{ MRI Findings } & Patients (No.) & $\begin{array}{c}\text { CT Findings Positive for } \\
\text { SC Dehiscence (No.) }\end{array}$ \\
\hline Negative for SC dehiscence & $90(80.4 \%)$ & 0 \\
Positive for SC dehiscence or inconclusive & $22(19.6 \%)$ & 8 \\
SC dehiscence & $13(11.6 \%)$ & 8 \\
Excluded SC & $8(7.1 \%)$ & 0 \\
Both SC dehiscence and excluded SC & $1(0.9 \%)$ & 0 \\
\hline
\end{tabular}

a One patient had simultaneously a SC dehiscence demonstrated on MRI and another SC excluded on the other side due to artifacts.

Table 5: Dehiscence measurement in FIESTA MRI and highresolution CT

\begin{tabular}{lcc}
\hline $\begin{array}{c}\text { Cases of Superior/Posterior } \\
\text { Semicircular Canal Dehiscence }\end{array}$ & MRI (mm) & CT (mm) \\
\hline On MRI and CT & & \\
SSCD1 & 5.1 & 3.6 \\
SSCD2 & 3.2 & 1 \\
SSCD3 & 7 & 3.8 \\
SSCD4 & 2.9 & 2,5 \\
SSCD5 & 3.3 & 2 \\
SSCD6 & 5 & 2.8 \\
SSCD7 & 1.7 & 2.5 \\
SSCD8 & 2.6 & 3.5 \\
SSCD9 & 4.6 & 3.7 \\
SSCD10 & 4.3 & 1.5 \\
SSCD11 & 4.3 & 1 \\
PSCD1 & 1,5 & 1.5 \\
Mean dehiscence length (mm) & $3.8^{\mathrm{a}}$ & 2.5 \\
P value & .009 & \\
On FIESTA only & & No dehiscence \\
SSCD12 & 4.1 & No dehiscence \\
SSCD13 & 4.4 & No dehiscence \\
SSCD14 & 2.3 & No dehiscence \\
SSCD15 & 2.6 & No dehiscence \\
SSCD16 & 4.2 & No dehiscence \\
SSCD17 & 1.1 & No dehiscence \\
SSCD18 & 4.2 & No dehiscence \\
PSCD2 & 1.5 & \\
PSCD3 & 1.7 & Nohiscence \\
Mean dehiscence length (mm) & $2.9^{\mathrm{a}}$ & \\
\hline
\end{tabular}

Note:- SSCD indicates superior semicircular canal dehiscence; $P S C D$, posterior semicircular canal dehiscence.

a Mean dehiscence length on FIESTA when present on CT was $3.9 \mathrm{~mm}$. Mean dehiscence length on FIESTA when absent on CT was $2.9 \mathrm{~mm}$. The difference between these 2 values is not statistically significant $(P=.18)$. were excluded. Of these same 448 SCs, MR imaging showed 21 SC dehiscence cases (14 patients) in 428 canals analyzable; 20 were excluded. The aggregate SC dehiscence incidence on CT was 2.7\% (12/ 448 SCs). The aggregate SC dehiscence incidence on MR imaging was 4.7\% (21/448 SCs).

Of 428 SCs available for analysis on both MR imaging and CT, MR imaging showed an absence of SC dehiscence in $95.1 \%$ (407/428 SCs). Including the excluded SCs, MR imaging showed an overall absence of SC dehiscence in $90.8 \%$ (407/448 SCs). MR imaging showed no SC dehiscence in 90 of the 112 patients $(80.4 \%)$ (Table 4$)$. The remaining 22 patients (19.6\%) had either at least $1 \mathrm{SC}$ dehiscence seen on MR imaging or $1 \mathrm{SC}$ excluded on MR imaging (13 patients with at least 1 SC dehiscence, 8 patients with at least $1 \mathrm{SC}$ excluded, 1 patient with both a SC dehiscence and another SC excluded).

\section{Dehiscence Measurement}

Table 5 details the measurement of dehiscence in our cases positive for SC dehiscence, either with CT or MR imaging. For FIESTA dehiscence measurements, we separately analyzed cases in which a dehiscence was seen on CT and cases in which no dehiscence was seen on CT. The mean dehiscence measurement on CT (12 cases, 11 SSCs and 1 PSC) was $2.5 \mathrm{~mm}$ (95\% CI, 1.8-3.1 mm). For these true-positive cases, the corresponding mean dehiscence length on FIESTA was $3.8 \mathrm{~mm}$ (95\% CI, $2.8-4.8 \mathrm{~mm}$ ). An example of a measurement is shown in Fig 5 . The difference in dehiscence measurement between MR imaging and CT was significant $(P=.009$, paired $t$ test).

The mean dehiscence value on MR imaging when there was no dehiscence seen on CT ( 9 cases, 7 SSCs and 2 PSCs) was $2.9 \mathrm{~mm}$ (95\% CI, $2.0-3.8 \mathrm{~mm})$. Although this value is $23 \%$ lower $(2.9$ versus $3.8 \mathrm{~mm})$, there was no significant statistical difference $(P=$ $.18, t$ test unpaired) in the mean dehiscence as measured on FIESTA MR imaging between those cases with dehiscence demonstrated on CT and those cases with no dehiscence demonstrated on CT.

\section{DISCUSSION}

Fully refocused steady-state gradient-echo MR images such as FIESTA (other manufacturer-specific acronyms include "constructive interference in steady state," "b-FFE," and "True FISP" ${ }^{2}$ ) offer a high signal-to-noise ratio that allows, within a reasonable time, submillimeter imaging and subsequent high-quality multiplanar reconstructions. FIESTA and similar sequences are typically included in MR imaging protocols to evaluate the internal auditory canal content for vestibular schwannoma because the high contrast between fluids and soft-tissue common to all steady 

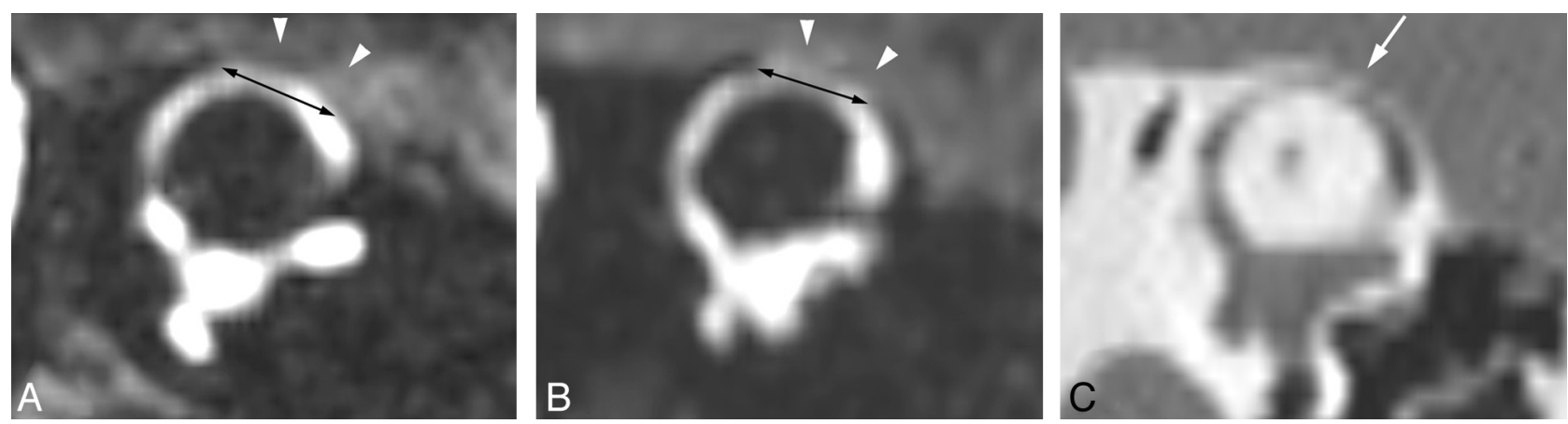

FIG 5. A 43-year-old woman with right-sided hearing loss and dizziness underwent MR imaging and CT twice within 9 months. The first FIESTA acquisition performed at 1.5T (A) shows the absence of a low-signal-intensity rim around the left SSC, suggesting dehiscence (arrowheads). At 3T (B), FIESTA again demonstrates clear absence of a low-signal-intensity rim around the SSC, suggesting dehiscence (arrowheads). The bone defect is measured at $4.4 \mathrm{~mm}$ on the $1.5 \mathrm{~T}$ acquisition ( $A$, black double-head arrow) and $4.2 \mathrm{~mm}$ on the $3 \mathrm{~T}$ acquisition ( $B$, black double-head arrow). Both 1.5T and 3T FIESTA acquisitions suggest SSC dehiscence. The small difference in measurement may be due to differences in magnetic susceptibility leading to greater conspicuity of bone coverage on the 3T FIESTA acquisition at both ends of the presumed dehiscence. In this case, $\mathrm{CT}(C)$ demonstrates a very thin but intact bone covering the SSC, excluding dehiscence (C, arrow).

state free precession sequences, combined with high spatial resolution, can be advantageously used to evaluate the fluid-filled spaces of the inner ear.

In this sequence, contrast is a function of the T2/T1 ratio in the target tissue, and tissues with long T2 values will usually have increased signal. ${ }^{11}$ As a result, fluid in the membranous labyrinth has high signal, whereas cortical bone has null signal because of low proton density and very short $\mathrm{T} 1$ and $\mathrm{T} 2 .{ }^{15}$ These features result in very high contrast between the membranous labyrinth and the surrounding petrous bone. FIESTA is theoretically wellsuited for the evaluation of SC dehiscence by enabling discrimination of very thin bone coverage between the membranous labyrinth and CSF, which is essential in the diagnosis of SC dehiscence. ${ }^{16}$

In our series, every SC dehiscence seen with CT was demonstrated on FIESTA, yielding a sensitivity of $100 \%$ in the FIESTA sequence compared with CT. FIESTA did, however, overestimate the presence of a dehiscence in 9 cases, with 21 cases of SC dehiscence seen on MR imaging and only 12 of them confirmed by CT. FIESTA depiction of bone coverage was less consistent than CT, leading to false-positive cases. In some instances, bony coverage of the semicircular canal is clearly so thin that signal loss is not perceivable on MR imaging, yet there was enough mineralization in it to be judged intact on CT. CT can demonstrate bone as thin as 0.1 $\mathrm{mm},{ }^{14}$ but it also may overestimate SC dehiscence by as much as a factor of 10 compared with anatomic-pathologic correlation studies. ${ }^{17}$

In evaluating the utility of FIESTA as a screening tool for SC dehiscence exclusion, the extreme sensitivity of this sequence for SC dehiscence despite a low positive predictive value appears to be an advantage. Our data show that a negative MR imaging finding effectively rules out SC dehiscence, with no case of dehiscence overlooked in our series. This result could have a significant impact on the imaging investigation of patients with vestibulocochlear signs and symptoms by reducing the need for temporal bone CT (by $>80 \%$ of the patients in our series) in patients presenting with vestibulocochlear symptoms that could be due to SC dehiscence but with other retrocochlear pathologies in the differential diagnosis; this would decrease subsequent radiation exposure and also cost.
Conversely, CT is still mandatory to confirm dehiscence in cases in which it is suspected on FIESTA, due to false-positive cases and subsequent low positive predictive value.

We also measured the length of the observed dehiscences because neurotologists use this information to decide whether they will operate. It has been recently demonstrated that dehiscence of $\geq 2.5 \mathrm{~mm}$ on CT is associated with significantly more vestibulocochlear symptoms than smaller dehiscences, ${ }^{18}$ perhaps because a relatively high number of small dehiscences seen on CT are probably not correlated with a real anatomic dehiscence. ${ }^{17}$ When SC dehiscence was seen, the measure of the dehiscence obtained with FIESTA was, on average, $>50 \%$ higher than the CT value, congruent with the assumption that FIESTA is less sensitive than CT in depicting very thin bone coverage; because of this difference, the 2.5-mm threshold used with CT cannot be transposed to FIESTA. When we compared the values of the dehiscence measurement on FIESTA between cases with and without dehiscence confirmed on CT, there was no significant difference, though we obtained a higher mean value of the dehiscence measured on FIESTA when there was a dehiscence seen on CT.

\section{Limitations}

The occurrence of SSC dehiscence and PSC dehiscence was 5.1\% and $0.5 \%$ in our population, which is the expected incidence range in patients referred for vestibulocochlear symptoms ${ }^{19}$ or anatomic-radiologic studies, ${ }^{17}$ but lower than a more recent study by using equivalent CT technology. ${ }^{20}$ This relatively low SSC and PSC dehiscence incidence leads to wide 95\% confidence interval results for sensitivity and positive predictive value. Specificity and negative predictive value confidence intervals are much smaller but will also require confirmation with a larger population study. The incidence of SC dehiscence in the general asymptomatic population remains unknown. ${ }^{21}$ This study did not evaluate lateral semicircular canal dehiscence, but that entity is more commonly associated with cholesteatoma. ${ }^{22,23}$

Some of the conditions that could result in a false-negative on FIESTA include banding artifacts, overlying blood vessels, and pneumocephalus. Banding artifacts due to field inhomogeneity are intrinsic to the FIESTA acquisition and produce curved hypointense lines. Banding artifacts were always well-recognized in 
our cases, rarely occurred exactly on the SC, and were not a problem for the analysis in any of our cases, but these are a potential pitfall. The constructive interference in steady state acquisition design includes 2 balanced steady state free precession runs with different flip angles to specifically address the banding artifacts problem but at a cost of a longer acquisition time. ${ }^{12}$ Vascular structures lying just over a dehiscent SSC could potentially be mistaken for normal bone coverage, though the vessel should then be easily recognized on adjacent sections. Similarly, pneumocephalus that gives marked hypointense signal could theoretically mimic bone but would not be expected on MR images obtained in a healthy outpatient population. Temporal bone erosive processes such as cholesteatoma, glomus tumor, or metastasis can produce SC dehiscence by direct bone lysis, but these pathologies present as mass lesions that should be easily recognized on MR imaging and/or CT.

Finally, another potential limitation is our use of both $1.5 \mathrm{~T}$ and 3T MR imaging scanners. Although there was no apparent difference in data distribution and results, one could speculate that with a larger study population, very thin bony coverage might be more conspicuous on 3T FIESTA.

\section{CONCLUSIONS}

FIESTA reliably excludes SC dehiscence with a negative predictive value of $100 \%$ and therefore can be used as an effective screening tool in the evaluation of patients with vestibulocochlear symptoms. Due to a low positive predictive value for SC dehiscence, only patients who are thought to be positive for SC dehiscence should proceed to temporal bone CT in the absence of other indications for CT scanning, to determine whether true SC dehiscence exists. In our patient population, $80 \%$ of patients would have been spared undergoing CT, with consequent prevention of exposure to radiation and significant cost reduction in the work-up for SC dehiscence.

Disclosures: Timothy L. Larson-Ownership Interest: Seattle Radiologists, Comments: partner in my private practice.

\section{REFERENCES}

1. Minor LB, Solomon D, Zinreich JS, et al. Sound- and/or pressureinduced vertigo due to bone dehiscence of the superior semicircular canal. Arch Otolaryngol Head Neck Surg 1998;124:249-58

2. Tullio P. Das Ohr Und Die Entstehung Der Sprache Und Schrift. Berlin, Germany: Urban \& Schwarzenberg; 1929:1-455

3. Krombach GA, Schmitz-Rode T, Haage P, et al. Semicircular canal dehiscence: comparison of T2-weighted turbo spin-echo MRI and CT. Neuroradiology 2004;46:326-31

4. Hirvonen TP, Carey JP, Liang CJ, et al. Superior canal dehiscence: mechanisms of pressure sensitivity in a chinchilla model. Arch Otolaryngol Head Neck Surg 2001;127:1331-36

5. Chi FL, Ren DD, Dai CF. Variety of audiologic manifestations in patients with superior semicircular canal dehiscence. Otol Neurotol 2010;31:2-10

6. Zhou G, Gopen Q, Poe DS. Clinical and diagnostic characterization of canal dehiscence syndrome: a great otologic mimicker. Otol Neurotol 2007;28:920-26

7. Krombach GA, DiMartino E, Schmitz-Rode T, et al. Posterior semicircular canal dehiscence: a morphologic cause of vertigo similar to superior semicircular canal dehiscence. Eur Radiol 2003;13:1444-50

8. Gopen Q, Zhou G, Poe D, et al. Posterior semicircular canal dehiscence: first reported case series. Otol Neurotol 2010;31:339-44

9. Torizuka T, Hayakawa K, Satoh Y, et al. High-resolution CT of the temporal bone: a modified baseline. Radiology 1992;184:109-11

10. Hatipoglu HG, Durakoglugil T, Ciliz D, et al. Comparison of FSE T2W and 3D FIESTA sequences in the evaluation of posterior fossa cranial nerves with MR cisternography. Diagn Interv Radiol 2007;13:56-60

11. Scheffler K, Lehnhardt S. Principles and applications of balanced SSFP techniques. Eur Radiol 2003;13:2409-18

12. Chavhan GB, Babyn PS, Jankharia BG, et al. Steady-state MR imaging sequences: physics, classification, and clinical applications. $R a$ diographics 2008;28:1147-60

13. Branstetter BF, Harrigal C, Escott EJ, et al. Superior semicircular canal dehiscence: oblique reformatted CT images for diagnosis. $\mathrm{Ra}$ diology 2006;238:938-42

14. Belden CJ, Weg N, Minor LB, et al. CT evaluation of bone dehiscence of the superior semicircular canal as a cause of sound- and/or pressure-induced vertigo. Radiology 2003;226:337-43

15. Techawiboonwong A, Song HK, Wehrli FW. In vivo MRI of submillisecond $\mathrm{T}(2)$ species with two-dimensional and three-dimensional radial sequences and applications to the measurement of cortical bone water. NMR Biomed 2008;21:59-70

16. Curtin HD. Superior semicircular canal dehiscence syndrome and multi-detector row CT. Radiology 2003;226:312-14

17. Crovetto M, Whyte J, Rodriguez OM, et al. Anatomo-radiological study of the superior semicircular canal dehiscence, radiological considerations of superior and posterior semicircular canals. Eur J Radiol 2010;76:167-172

18. Pfammatter A, Darrouzet V, Gartner M, et al. A superior semicircular canal dehiscence syndrome multicenter study: is there an association between size and symptoms? Otol Neurotol 2010;31:447-54

19. Picavet V, Govaere E, Forton G. Superior semicircular canal dehiscence: prevalence in a population with clinical suspected otosclerosis-type hearing loss. B-ENT 2009;5:83-88

20. Stimmer H, Hamann KH, Zeiter S, et al Semicircular canal dehiscence in HR multislice computed tomography: distribution, frequency, and clinical relevance. Eur Arch Otorhinolaryngol 2012;269:475-80

21. Loke SC, Goh JP. Incidence of semicircular canal dehiscence in Singapore. Br J Radiol 2009;82:371-73

22. Bassim MK, Patel KG, Buchman CA. Lateral semicircular canal dehiscence. Otol Neurotol 2007;28:1155-56

23. Zhang YB, Dai CF, Sha Y. Sound-induced vertigo due to bone dehiscence of the lateral semicircular canal. Eur Arch Otorhinolaryngol 2010;267:1319-21 